\section{Effect of COVID-19 on presentations of decompensated liver disease in Scotland}

To cite: Manship T, Brennan PN, Campbell I, et al. Effect of COVID-19 on presentations of decompensated liver disease in Scotland. BMJ Open Gastro 2022;9:e000795. doi:10.1136/ bmjgast-2021-000795

\section{- Additional supplemental material is published online only. To view, please visit the journal online (http://dx.doi. org/10.1136/bmjgast-2021- 000795).}

Received 3 0ctober 2021 Accepted 17 December 2021
Check for updates

\section{(C) Author(s) (or their} employer(s)) 2022. Re-use permitted under CC BY. Published by BMJ.

For numbered affiliations see end of article.

Correspondence to Dr Paul N Brennan; p.z.brennan@dundee.ac.uk

\section{ABSTRACT}

Background and aims SARS-CoV-2 and consequent pandemic has presented unique challenges. Beyond the direct COVID-related mortality in those with liver disease, we sought to determine the effect of lockdown on people with liver disease in Scotland. The effect of lockdown on those with alcohol-related disease is of interest; and whether there were associated implications for a change in alcohol intake and consequent presentations with decompensated disease.

Methods We performed a retrospective analysis of patients admitted to seven Scottish hospitals with a history of liver disease between 1 April and 30 April 2020 and compared across the same time in 2017, 2018 and 2019. We also repeated an intermediate assessment based on a single centre to examine for delayed effects between 1 April and 31 July 2020.

Results We found that results and outcomes for patients admitted in 2020 were similar to those in previous years in terms of morbidity, mortality, and length of stay. In the Scotland-wide cohort: admission MELD (Model for Endstage Liver Disease) (16 (12-22) vs 15 (12-19); $p=0.141)$, inpatient mortality ((10.9\% vs $8.6 \%) ; p=0.499)$ and length of stay (8 days (4-15) vs 7 days (4-13); $p=0.140)$. In the Edinburgh cohort: admission MELD (17 (12-23) vs 17 (13-21); $p=0.805)$, inpatient mortality $((13.7 \%$ vs $10.1 \%$; $\mathrm{p}=0.373)$ and length of stay (7 days (4-14) vs 7 days $(3.5-14) ; p=0.525))$.

Conclusion This assessment of immediate and mediumterm lockdown impacts on those with chronic liver disease suggested a minimal effect on the presentation of decompensated liver disease to secondary care.

\section{INTRODUCTION}

SARS-CoV-2 and consequent COVID-19 pandemic has presented unique challenges across the globe. Most countries introduced severe restrictions on individual freedoms in an attempt to reduce COVID-19-associated mortality. Persons with liver disease represent a diverse spectrum of aetiologies; however, they share a relative immune dysfunctional state. Recently, a large cohort study has been published, which suggested that SARS-CoV2 mortality in all-cause liver disease was $32 \%$
Summary box

What is already known about this subject?

- The COVID-19 pandemic has presented significant challenges to the provision of medical care for patients with liver disease in Scotland.

What are the new findings?

- Our data would suggest that in the short to medium term there did not appear to be any demonstrable adverse outcome in patients with chronic liver disease in Scotland.

- This finding included those with alcohol-related liver disease, which has been shown to have increased mortality in England and Wales over the course of the pandemic.

How might it impact on clinical practice in the foreseeable future?

- While our primary focus related to the initial lockdown period of 2020 was reassuring, it will be important to see the latter effect of further lockdown measures.

- Future work aimed at assessing the unknown longer term implications of lockdown and the changes in healthcare delivery approaches post-pandemic are paramount in determining future-proofing strategies.

- This is particularly pressing given the persistent threat posed by further waves of endemic SARSCoV-2 variants.

(71\% respiratory failure) ${ }^{1}$ which has enormous implications for such patients.

COVID-19 lockdown began in Scotland on 23 March 2020. This led to a closure of non-essential services including pubs, restaurants, and shops. All non-essential workers were encouraged to work from home. ${ }^{2}$ There was a shifting of the National Health Service (NHS) care with all elective work cancelled and the focus becoming inpatient, emergency care with increasing emphasis on COVID-19infected individuals.

An Alcohol Change UK survey ${ }^{3}$ reported that during the UK lockdown (24 March 
2020-4 July 2020) alcohol consumption was reduced in the majority but $20 \%$ of respondents increased their alcohol intake. In those patients with a history of alcohol misuse, 24\% had increased alcohol consumption and $17 \%$ relapsed to drinking. ${ }^{4}$ Scottish Public Health Observatory ${ }^{5}$ data show that Scotland has one of the highest rates of chronic liver disease (CLD) deaths in Europe. The rate in Scotland in 2016 was 16.8 per 100000 . This is in comparison with the Netherlands where CLD mortality was 3.8 per 100000 . Alcohol-related liver disease (ArLD) is the most common cause of CLD in Scotland. Although difficult to estimate the number of patients in the community with ArLD, due to undiagnosed disease, in 2018/2019 there were 129.3 per 100000 hospital stays with CLD due to alcohol. In 2018, Scottish patients with CLD were over four times as likely to die if they had ArLD when compared with all other causes of liver disease ${ }^{5}$ $(13.1 / 100000$ vs $3.1 / 100000)$.

Mahmud et at showed in a large study of veterans in the USA with a history of CLD that they were less likely to be admitted to hospital and were more unwell when admitted early in the COVID-19 pandemic when compared with previous years. Gaspar et $a l^{7}$ found contrasting data during the Portuguese state of emergency.

In view of Scotland's high rate of CLD due to alcohol and the reported increase in alcohol consumption during lockdown, our aim was to describe first the effect of lockdown on all liver-related admissions in the short and medium terms and second the outcome of patients with ArLD in the medium term.

\section{MATERIALS AND METHODS}

To determine the immediate effect of COVID-19 lockdown, data were collected from across Scotland on patients admitted with liver disease. Patients admitted to seven Scottish hospitals with a history of liver disease under the care of a gastroenterologist on dedicated gastroenterology or hepatology wards between 1 April and 30 April 2020 were included and compared with those admitted in the same period in 2017, 2018 and 2019. The Scottish sites were Royal Infirmary of Edinburgh (RIE) and Western General Hospital, Edinburgh; St John's Hospital, Livingston; Glasgow Royal Infirmary and Queen Elizabeth University Hospital, Glasgow; Ninewells Hospital, Dundee; Hairmyres Hospital, Lanarkshire and Dumfries and Galloway Royal Infirmary.

To assess the medium-term effect of the healthcare reorganisation during the pandemic, data were collected on patients admitted to the RIE with a history of liver disease under the care of a hepatologist. RIE is a large, tertiary hepatology centre. Patients admitted between 1 April 2020 and 31 July 2020 were compared with those admitted in the same period in 2017, 2018 and 2019.

We included any patient admitted under the care of a gastroenterologist or a hepatologist with a history of CLD, regardless of admission diagnosis. We excluded those patients under the age of 18 , patients admitted electively and those with a history of liver transplantation. No patients were admitted with COVID-19 infection at the time of writing this manuscript.

Variables collected were patient demographics, reason for admission, disease characteristics (history of hepatocellular carcinoma (HCC), history of ascites, presence of oesophageal varices and history of hepatic encephalopathy (HE)). Information on aetiology of liver disease was also collected and was split into ArLD and non-ArLD. Diagnosis was as determined by the patient's usual clinician. Where the patient had more than one diagnosis, the primary diagnosis as per their usual clinician was used. Severity of liver disease on admission was defined by United Kingdom End-stage Liver Disease (UKELD) ${ }^{8}$ and Model for End-stage Liver Disease $\left(\right.$ MELD $^{9}$ scores. Data were collected on socioeconomic deprivation using the Scottish Index of Multiple Deprivation (SIMD) (2020 update). This is a composite score of multiple factors (including income, employment, health, education, access to services, crime and housing) to determine deprivation in different parts of Scotland. ${ }^{10}$ The reason for admission was condensed into 'decompensation' (jaundice, ascites, gastrointestinal bleeding, HE and spontaneous bacterial peritonitis) and 'non-decompensation' (all other diagnoses) for analysis. Outcome measures were inpatient mortality and length of stay.

Demographics and baseline characteristics of the cohort were compared using the Pearson $\chi^{2}$ test for categorical variables. Continuous variables were compared using the Student's t-test (parametric) or the MannWhitney U test (non-parametric). Categorical variables were recorded as numbers (percentages) and continuous variables as median (IQR). Data were analysed using SPSS V.24. Significant values were determined by a $p$ value of equal to or less than 0.05 .

\section{RESULTS}

\section{Immediate effect of lockdown: April 2020 (seven Scottish} sites)

Three hundred forty-eight patients were admitted to seven hospitals in April in the years 2017 ( $\mathrm{n}=123), 2018$ $(n=116)$ and $2019(n=109)$ in comparison with 111 in the same period in 2020 (table 1). Patient demographics and levels of socioeconomic deprivation were similar in the two time cohorts. Most admission blood variables were also similar between the two groups; however, serum sodium was significantly lower in the pre-COVID (20172019) cohort compared with the 2020 cohort (135 (130138) vs 137 (132-140); $\mathrm{p}=0.005)$. Consequently, UKELD score in the pre-COVID cohort was significantly higher (56 (52-60) vs $54(50-60) ; \mathrm{p}=0.005)$. No difference in the pre-2016 MELD (without sodium) was observed. Characteristics of cirrhosis were also similar apart from the presence of HCC which was more prevalent in the pre-COVID cohort $(7.2 \%$ vs $1.8 \%$; $\mathrm{p}=0.036)$. Aetiology of liver disease was also similar between the two groups (when comparing ArLD with non-ArLD diagnoses). 
Table 1 A comparison of patients admitted between 1 April and 30 April 2020 to seven sites across Scotland with a history of chronic liver disease

\begin{tabular}{|c|c|c|c|c|c|}
\hline & & Reference range & $\begin{array}{l}2017-2019 \\
(\mathrm{n}=348) \\
\text { Pre-COVID }\end{array}$ & $\begin{array}{l}2020 \\
(n=111) \\
\text { Lockdown }\end{array}$ & $P$ value \\
\hline \multirow[t]{2}{*}{ Patient demographics } & Age (years) & & $57(49-65)$ & $59(47-68)$ & 0.879 \\
\hline & Gender (male) (\%) & & $208(59.8 \%)$ & $59(53.2 \%)$ & 0.218 \\
\hline \multirow[t]{5}{*}{ SIMD } & 1 (most deprived) & & $161(46.3 \%)$ & $46(41.4 \%)$ & 0.422 \\
\hline & 2 & & $56(16.1 \%)$ & $21(18.9 \%)$ & \\
\hline & 3 & & $45(13.0 \%)$ & $15(13.5 \%)$ & \\
\hline & 4 & & $54(15.6 \%)$ & $13(11.7 \%)$ & \\
\hline & 5 (least deprived) & & $32(9.2 \%)$ & $16(14.5 \%)$ & \\
\hline \multirow[t]{21}{*}{ Admission characteristics } & $\mathrm{Hb}(\mathrm{g} / \mathrm{L})$ & $115-165$ & $105(82-123)$ & $104.5(86.5-127)$ & 0.553 \\
\hline & $\operatorname{WCC}\left(\times 10^{9}\right)^{*}$ & $4-11$ & $7.4(4.9-10.7)$ & $7.6(5.2-11.5)$ & 0.591 \\
\hline & Platelets $\left(\times 10^{9}\right)$ & $150-400$ & $114(75-174)$ & $121(88-185)$ & 0.495 \\
\hline & $\mathrm{PT}(\mathrm{s})$ & $10.5-13.4$ & $17(14-21)$ & $17(14-20)$ & 0.378 \\
\hline & Urea (mmol/L) & $2.5-6.6$ & $5.0(3.1-8.6)$ & $4.9(3.3-8.6)$ & 0.542 \\
\hline & Sodium (mmol/L) & $135-145$ & $135(130-138)$ & $137(132-140)$ & 0.005 \\
\hline & Creatinine (mmol/L) & $50-98$ & $69(56-97)$ & $72(51-103)$ & 0.894 \\
\hline & Potassium (mmol/L) & $3.6-5$ & $4.1(3.6-4.5)$ & $4.1(3.5-4.5)$ & 0.711 \\
\hline & Bilirubin (mmol/L) & $3-21$ & $50(24-113)$ & $42(18-101)$ & 0.097 \\
\hline & ALT (U/L) & $10-50$ & $29(20-47)$ & $32(23-45)$ & 0.568 \\
\hline & ALP (U/L) & $40-125$ & $157(111-230)$ & $141(104-202)$ & 0.071 \\
\hline & Albumin (g/L) & $36-47$ & $27(22-31)$ & $28(23-33)$ & 0.082 \\
\hline & MELD & & $16(12-22)$ & $15(12-19)$ & 0.224 \\
\hline & UKELD & & $56(52-60)$ & $54(50-60)$ & 0.005 \\
\hline & Admission hospital & GRI & $83(23.9 \%)$ & $23(20.7 \%)$ & \\
\hline & & QEUH & $77(22.1 \%)$ & $34(30.6 \%)$ & \\
\hline & & RIE & $76(21.8 \%)$ & $22(19.8 \%)$ & \\
\hline & & Lanarkshire & $43(12.4 \%)$ & $10(9.0 \%)$ & \\
\hline & & Ninewells & $41(11.8 \%)$ & $11(9.9 \%)$ & \\
\hline & & DGRI & $22(6.3 \%)$ & 7 (6.3\%) & \\
\hline & & WGH & $6(1.7 \%)$ & $4(3.6 \%)$ & \\
\hline \multirow[t]{2}{*}{ Inpatient outcomes } & Length of stay (days) & & $8(4-15)$ & $7(4-13)$ & 0.131 \\
\hline & Inpatient mortality (\%) & & $36(10.3 \%)$ & $9(8.1 \%)$ & 0.490 \\
\hline \multirow[t]{4}{*}{ Disease characteristics } & HCC (\%) & & $25(7.2 \%)$ & $2(1.8 \%)$ & 0.036 \\
\hline & Ascites (\%) & & $187(53.7 \%)$ & $51(45.9 \%)$ & 0.953 \\
\hline & Varices (\%)† & & $206(67.5 \%)$ & $57.3(60 \%)$ & 0.074 \\
\hline & HE (\%) & & $110(31.6 \%)$ & $42(37.8 \%)$ & 0.225 \\
\hline \multirow[t]{2}{*}{ Aetiology of liver disease } & ArLD (\%) & & $263(75.6 \%)$ & $82(73.9 \%)$ & 0.718 \\
\hline & Non-ArLD & & $85(24.4 \%)$ & $29(26.1 \%)$ & \\
\hline \multirow[t]{2}{*}{ Reason for admission } & Decompensation (\%) & & $267(76.7 \%)$ & $80(72.1 \%)$ & 0.320 \\
\hline & Non-decompensation (\%) & & $81(23.3 \%)$ & $31(27.9 \%)$ & \\
\hline
\end{tabular}

All values are median/IQR unless otherwise stated.

*Patients admitted to one trust did not have white cell breakdown results recorded; therefore, this information was excluded from this part of the analysis.

†Those with no previous endoscopies were excluded $(n=65)$.

ALP, alkaline phosphatase; ALT, alanine transaminase; ArLD, alcohol-related liver disease; DGRI, Dumfries and Galloway Royal Infirmary; GRI, Glasgow Royal Infirmary; Hb, haemoglobin; HCC, hepatocellular carcinoma; MELD, Modified score for End-stage Liver Disease; PT, prothrombin time; QEUH, Queen Elizabeth University Hospital; RIE, Royal Infirmary of Edinburgh; SIMD, Scottish Index of Multiple Deprivation; UKELD, United Kingdom End-stage Liver Disease score; WCC, white cell count; WGH, Western General Hospital. 
Outcome data were also similar between the two groups. Median length of stay was 8 days (4-15) in the pre-COVID cohort compared with 7 days (4-13) in the COVID-19 cohort $(\mathrm{p}=0.131)$. Inpatient mortality was also not statistically different between the two groups $(10.3 \%$ $(n=36)$ vs $8.1 \%(n=9) ; p=0.490)$.

\section{Medium-term effect of lockdown: April-July 2020 (RIE)}

To determine if there was a medium-term effect on COVID-19 healthcare reorganisation, 284 patients admitted with CLD in the 4-month period in 2017 ( $\mathrm{n}=82)$, $2018(\mathrm{n}=86)$ and $2019(\mathrm{n}=116)$ were compared with 89 in 2020 to the RIE (table 2). Patient demographics and social deprivation scoring were similar. Blood results on admission were also similar apart from serum sodium which was significantly lower in the pre-COVID cohort (134 (130-138) vs 136 (131-139); p=0.042). Severity of liver disease scores (MELD and UKELD) were similar between the two groups in contrast with previous. Disease

Table 2 A comparison of patients admitted between April and July 2017-2019 to those in 2020 to the Royal Infirmary, Edinburgh with a history of chronic liver disease

\begin{tabular}{|c|c|c|c|c|c|}
\hline & & Reference range & $\begin{array}{l}2017-2019(n=284) \\
\text { Pre-COVID }\end{array}$ & $\begin{array}{l}2020(n=89) \\
\text { Lockdown }\end{array}$ & $P$ value \\
\hline \multirow[t]{2}{*}{ Patient demographics } & Age (years) & & $57.1(49-67)$ & $58.0(48-68)$ & 0.912 \\
\hline & Gender (male) (\%) & & $46(55.4 \%)$ & $175(61.6 \%)$ & 0.096 \\
\hline \multirow[t]{5}{*}{ SIMD } & 1 (most deprived) & & $70(24.7 \%)$ & $22(24.7 \%)$ & 0.791 \\
\hline & 2 & & $65(23.0 \%)$ & $19(21.3 \%)$ & \\
\hline & 3 & & $51(18.0 \%)$ & $17(19.1 \%)$ & \\
\hline & 4 & & $43(15.2 \%)$ & $12(13.5 \%)$ & \\
\hline & 5 (least deprived) & & $54(19.1 \%)$ & $19(21.4 \%)$ & \\
\hline \multirow[t]{15}{*}{ Admission characteristics } & Haemoglobin (g/L) & $115-165$ & $108(90-122)$ & $106(83.5-121.5)$ & 0.494 \\
\hline & WCC $\left(\times 10^{9}\right)$ & $4-11$ & $8.0(5.9-11.5)$ & $7.6(5.3-11.8)$ & 0.401 \\
\hline & Neutrophils $\left(\times 10^{9}\right)$ & $2-7.5$ & $5.54(3.90-8.73)$ & $4.75(3.25-8.15)$ & 0.241 \\
\hline & Lymphocytes $\left(\times 10^{9}\right)$ & $1.5-4.5$ & $1.11(0.73-1.65)$ & $1.16(0.78-1.78)$ & 0.388 \\
\hline & Platelets $\left(\times 10^{9}\right)$ & $150-400$ & $114(75-174)$ & $124(80-175)$ & 0.442 \\
\hline & $\mathrm{PT}(\mathrm{s})$ & $10.5-13.5$ & $17(15-22)$ & $17(15-20)$ & 0.469 \\
\hline & Urea (mmol/L) & $2.5-6.6$ & $5.3(3.5-10.2)$ & $5.5(3.1-10.2)$ & 0.896 \\
\hline & Sodium (mmol/L) & $135-145$ & $134(130-138)$ & $136(131-139)$ & 0.042 \\
\hline & Creatinine (mmol/L) & $50-98$ & 73 (61-109) & $76(59-112)$ & 0.988 \\
\hline & Potassium (mmol/L) & $3.6-5$ & $4.0(3.6-4.4)$ & $4.0(3.5-4.5)$ & 0.980 \\
\hline & Bilirubin (mmol/L) & $3-21$ & $53(29-149)$ & $60(31-109)$ & 0.646 \\
\hline & $\operatorname{ALT}(\mathrm{U} / \mathrm{L})$ & $10-50$ & $32(20-54)$ & $34(22-54)$ & 0.533 \\
\hline & ALP (U/L) & $40-125$ & $178(116-256)$ & $164(124-210)$ & 0.179 \\
\hline & MELD & & $17(12-23)$ & $17(13-21)$ & 0.805 \\
\hline & UKELD & & $57(53-62)$ & $57(53-60)$ & 0.246 \\
\hline \multirow[t]{2}{*}{ Inpatient outcomes } & Length of stay (days) & & $7(4-14)$ & $7(3.5-14)$ & 0.525 \\
\hline & Inpatient mortality (\%) & & $39(13.7 \%)$ & $9(10.1 \%)$ & 0.373 \\
\hline \multirow[t]{4}{*}{ Disease characteristics } & $\operatorname{HCC}(\%)$ & & $26(9.2 \%)$ & $4(4.5 \%)$ & 0.158 \\
\hline & Ascites (\%) & & $177(62.3 \%)$ & $54(60.7 \%)$ & 0.780 \\
\hline & Varices $^{*}(\%)$ & & $173(62.0 \%)$ & $50(61.0 \%)$ & 0.866 \\
\hline & HE (\%) & & $102(35.9 \%)$ & $19(21.3 \%)$ & 0.010 \\
\hline \multirow[t]{2}{*}{ Aetiology of liver disease } & ARLD (\%) & & 237 (83.5\%) & $76(85.4 \%)$ & 0.663 \\
\hline & Non-ARLD & & 47 (16.5\%) & $13(14.6 \%)$ & \\
\hline \multirow[t]{2}{*}{ Reason for admission } & Decompensation (\%) & & $235(82.7 \%)$ & $63(70.8 \%)$ & 0.014 \\
\hline & Non-decompensation (\%) & & $49(17.3 \%)$ & $26(29.2 \%)$ & \\
\hline
\end{tabular}

All values are median/IQR unless otherwise stated.

*Those with no previous endoscopies were excluded ( $n=12)$.

ALP, alkaline phosphatase; ALT, alanine transaminase; ArLD, alcohol-related liver disease; HCC, hepatocellular carcinoma; MELD, Modified score for End-stage Liver Disease; PT, prothrombin time; UKELD, United Kingdom End-stage Liver Disease score; WCC, white cell count. 


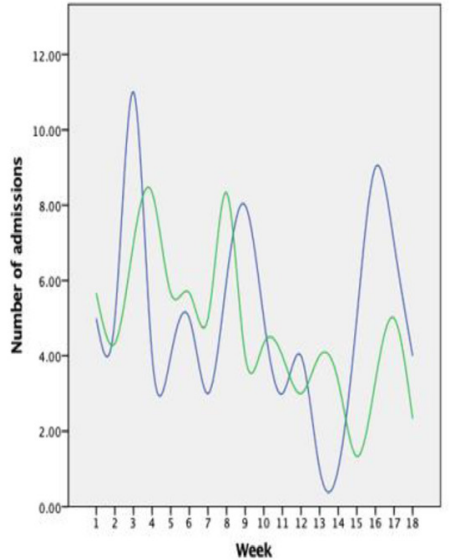

Key $2017-2019$ (mean):

2020:-

Figure 1 Weekly number of admissions in 2020 compared with the mean number of admissions in the previous 3 years.

characteristics were also similar between the two groups apart from the history of $\mathrm{HE}$ which was more prevalent in patients admitted in the pre-COVID cohort $(35.9 \%$ $(\mathrm{n}=102)$ vs $21.3 \% \quad(\mathrm{n}=19) ; \mathrm{p}=0.01)$. Aetiology of liver disease between the two groups was similar. Patients in the pre-COVID cohort were more likely to have been admitted with decompensated liver disease than in the COVID-19 cohort $(82.7 \% \quad(n=235)$ vs $70.8 \% \quad(n=63)$; $\mathrm{p}=0.014)$.

Outcome data were similar between the two groups. Median length of stay in both cohorts was 7 days $(p=0.525)$ (7 days (4-14) vs 7 days (3.5-14)). Inpatient mortality was not significantly different $(13.7 \%(n=39)$ vs $10.1 \%(n=9)$; $\mathrm{p}=0.373)$.

Although RIE is a large, tertiary referral centre for liver disease, with a transplant unit the vast major of these patients were using the centre as their local hospital $(93 \%)$ with very few coming from out of area $(7 \%)$.

We plotted the number of weekly admissions in the 18 weeks included in 2020 on the same graph as the mean number of admissions each week in 2017-2019 (figure 1). As can be seen, there are multiple points of crossover and therefore the weekly number of admissions are not significantly different.

As previously discussed, Scotland has a high rate of ArLD cirrhosis. Therefore, we performed a subgroup analysis as part of our medium-term cohort of patients admitted to RIE. We compared those with ArLD admitted in $2020(n=76 ; 85.4 \%$ of all patients admitted $)$ with those in the previous 3 years $(n=237 ; 83.4 \%$ of all patients admitted). The two groups were again similar, and no differences were found in patient demographics, severity scoring, length of stay or inpatient mortality (see online supplemental information). Patients admitted in the preCOVID cohort were, once again, found to have a significantly lower serum sodium on admission compared with those in the previous 3 years (133 (129-137) vs 136 (131-139); $\mathrm{p}=0.010)$. This then led to a higher UKELD in patients admitted in 2017-2019 in comparison with the COVID-19 cohort (58 (54-63) versus 57 (53-60); $\mathrm{p}=0.040)$ that was not replicated in MELD scores. Patients with a history of $\mathrm{HE}$ were more likely to be admitted in the pre-COVID cohort than those in the COVID-19 cohort $(36.3 \%$ vs $21.1 \% ; \mathrm{p}=0.014)$. No differences were found in the number of patients with other disease characteristics. No differences were seen in the reasons for admissions between the two groups.

Outcomes, once again, were found to be similar between the two groups. Median length of stay was 7 days in both groups ( 7 days (4-15) versus 7 days (4-14); $\mathrm{p}=0.821$ ). Inpatient mortality was $13.5 \%$ in the pre-COVID cohort and $6.6 \%$ in the COVID-19 cohort $(p=0.104)$.

\section{CONCLUSION}

Our data demonstrate no immediate or medium-term effect of NHS reconfiguration and lockdown in Scotland on numbers of admissions of patients with liver disease or adverse outcomes, including length of stay and inpatient mortality. There was no suggestion that patients were admitted with more severe or advanced liver disease or complications of their disease. Patient demographics and socioeconomic deprivation were no different between the two groups in the immediate lockdown period, in the medium term and in the ArLD subgroup. Overall, all blood parameters on admission were the same apart from serum sodium which reached a statistically significant difference but is of doubtful clinical relevance. Given the heavy weighting of serum sodium in the UKELD score, this is the likely reason for the higher scores in the immediate post-lockdown group and in the ArLD subgroup. No other components of the UKELD score were significantly different, and the original MELD score, which does not include serum sodium, were also no different.

On the whole, disease characteristics were the same between the two groups, although we did note higher rates of $\mathrm{HCC}$ in the pre-COVID April group and $\mathrm{HE}$ in the pre-COVID April-July and ARLD subgroup. These differences are difficult to explain but it could be related to restricted healthcare provision during COVID lockdown, that is, less surveillance for diagnosis of HCC and less support for people with known HE. Another possibility is the effect of social isolation from family and carers during the pandemic. Patients are commonly admitted with $\mathrm{HE}$ due to concerns from family and therefore reduced interactions could lead to a reduced number of admissions. In a study examining the impact of COVID-19 on the management of HCC, Amaddeo et $a l^{11}$ found that fewer patients were referred with index cases of HCC, and fewer had a first diagnosis of HCC during a 6-week period of the pandemic compared with 2019. This was likely related to the reduction in patient contact with health practitioners. Gandhi Met et $a l^{12}$ also demonstrated a reduction in the rates of HCC diagnosis $(26.7 \%)$ in an Asia-Pacific population between February and May 2020 compared with the previous year. Both these groups were focused on the diagnosis of HCC, but both demonstrated the reduced number of presentations 
to health services with HCC, similar to the data presented here.

In the short-term and medium-term cohorts, we found no difference in aetiology of liver disease when comparing patients with ArLD to all other patients. We had believed that patients with ArLD would be more prominent in the COVID-19 era cohorts due to increased alcohol intake during lockdown; however, as previously discussed, this seems to not be the case. Patients could be avoiding admissions due to concerns regarding COVID-19 and in turn there could be a delayed effect with no differences seen within the timeframe of this study.

Reasons for admission (decompensated disease vs non-decompensated disease) were also generally similar. In the medium-term cohort, there were more people admitted with decompensated disease in 2017-2019.

Patient outcomes, in this study both length of stay and inpatient mortality, are clearly the factors that impact patients most. Our study demonstrated no difference in length of stay or inpatient mortality in any of the three groups that we investigated. This is reassuring and demonstrates that, in both the short and medium terms, inpatient medical care has not been affected by the pandemic.

The decreasing number of CLD admissions in Scotland over the last few years should be considered. According to Scottish Public Health Observatory data, ${ }^{13}$ there were 11002 admissions (208.01 per 100000 population) to Scottish hospitals in the financial year 2016/2017 compared with 10917 (204.4 per 100 000) in 2017/2018 and 10378 (193.18 per 100000 ) in 2018/2019. This demonstrates around a 5\% reduction in hospital admissions. There has also been a reduction in the number of CLD-related deaths at the same time: from 890 in 2016 to 873 in 2018. It is possible that given the number of admissions had been decreasing pre-COVID that the pandemic has had minimal effect when comparing admissions in 2020 compared with the previous 3 years. This is also possible with inpatient mortality, although CLD deaths have decreased less significantly.

In May 2018, minimum unit pricing (MUP) for alcohol came into force at 50 pence per unit in Scotland. ${ }^{14}$ An analysis in 2021 showed a 4\%-5\% decrease in 'off-trade' alcohol sales (purchased from a shop rather than a pub or restaurant) in the 12 months following the implementation of MUP ${ }^{15}$ and analysis into how this interacts with hospital-related admissions and outcomes is ongoing. It is possible that this decrease in sales and presumed consumption of alcohol has led to fewer complications of ArLD and therefore a reduced number of admissions than would normally be expected and therefore altering the impact of the pandemic. This is important given most of the patients in both our cohorts had ArLD. A Public Health Scotland report, ${ }^{16}$ however, found that there was no significant change in alcohol consumption levels during the first lockdown compared with the same period the previous year. Although, this was felt to be due to an increase in 'off trade' drinking offset by a decrease in 'on-trade' drinking. There was also no significant increase in the number of weekdays with early drinking (before 17:00) or solitary drinking occasions in a week.

These results differ from the largest study to date in this area $(n=12467)$. Mahmud et at demonstrated almost 160 fewer admissions than would be expected in veterans with decompensated cirrhosis during the pandemic by difference-in-difference analysis. They also found that those admitted later in the pandemic had a higher admission MELD and shorter length of stay which they conclude is likely due to a combination of patients being encouraged to stay at home and patients avoiding hospital and therefore only presenting when more unwell. A reduction in the number of cirrhosis admissions was also seen in a tertiary Chinese centre ${ }^{17}$ during the height of lockdown (21 January 2020 to 31 March 2020) but this was a much smaller study $(n=414)$ than Mahmud. This pattern of reduced admissions but those admitted being more unwell has been shown in patients with heart failure ${ }^{18} 19$ and those with acute coronary syndromes. ${ }^{20}$

In contrast, Gaspar et al carried out a study in Portugal looking at trends in cirrhosis admissions in comparison to previous years $(n=40$ in 2020). They demonstrated a similar number of admissions, severity of liver disease parameters and length of stay. They also demonstrated no difference in in-hospital mortality but did find significantly reduced readmission rates in 2020. A German study by $\mathrm{Zu}$ Siederdissen $e t a l^{21}$ found that although there was a reduction in the number of emergency admissions to the hospital, there was no difference in the number of patients with liver cirrhosis nor a difference in the severity of liver disease at presentation nor 30-day mortality.

Our study clearly has several limitations. We were unable to gather data from across the whole of Scotland when looking at the initial month following COVID-19 lockdown. However, we did gather data from health boards with most liver-related admissions in recent years $^{13}$ (NHS Lothian, NHS Greater Glasgow and Clyde and NHS Lanarkshire). Our study also covers three of the four largest cities in Scotland (Glasgow, Edinburgh, and Dundee). Scotland had very low COVID-19 prevalence during the summer of 2020 (during June and July less than 30 cases $/$ day $^{22}$ with less than $1 \%$ of all tests positive) and normal services, such as outpatient clinics and elective admissions, started to resume. Therefore, given the snapshot interval (April 2020), it is possible that this was not long enough to effectively assess the impact.

Our analysis of the medium-term effects of lockdown covered a longer period (April-July 2020); however, it was only based at a single centre (RIE). This means that it does not have the breadth of looking at more Scottish sites. However, RIE is a large, tertiary referral centre for liver disease in Scotland, which is large enough to be representative of the wider population. As mirrored in the Scotland-wide data, NHS Lothian, where RIE is situated, had minimal cases in the summer months of 2020. Indeed, on only 1 day was there more than 10 recorded cases in a day in June (2 June 2020; 14 cases $^{22}$ ). 
It is, however, possible that the reduced admission rates due to decompensation in the Edinburgh cohort in 2020 could reflect delayed presentations. As outlined by Tapper et $a l,{ }^{23}$ normal services resuming following the first wave could lead to significantly increased demand due to previously delayed care and fear of seeking medical attention. This is corroborated by Toyoda et $a l^{24}$ who showed that liver clinic visits, hepatoma surveillance and diagnostic imaging in patients with cirrhosis decreased significantly during COVID-19 lockdowns in three medical centres. This will inevitably lead to a delay in the recognition of decompensation and the diagnosis of HCC. Blach et $a l^{25}$ also demonstrated the potential effects of reduced hepatitis $\mathrm{C}$ elimination due to the pandemic on excess HCC cases and excess liver related deaths between 2020 and 2030. Clearly, such changes could alter mediumterm inpatient length of stay and mortality, but have not been captured in our cohort given the relatively short follow-up period.

Our study only looked at patients admitted to gastroenterology or hepatology wards. It is possible that given the restructuring of hospital services during the pandemic, that patients were admitted to other clinical areas and managed by other medical specialties and therefore would not be included in this cohort. It is unlikely, however, that patients with decompensated disease, which comprises most patients, would be admitted under the care of another specialty without specialist gastroenterology support.

In conclusion, our data would suggest that the initial lockdown related to the COVID-19 pandemic had minimal effect on patients with CLD in Scotland. While the initial focus was on the first lockdown period, it will be important to determine the effect of further lockdowns as the pandemic continues, particularly as the country leaves a period of further restriction. Future work aimed at assessing the longer term implications of lockdown is paramount in future care provision in case of further pandemic threats.

\section{Author affiliations}

${ }^{1}$ Centre for Liver and Digestive Disorders, Royal Infirmary of Edinburgh, Edinburgh, UK

${ }^{2}$ Centre for Regenerative Medicine, The University of Edinburgh Edinburgh Medical School, Edinburgh, UK

${ }^{3}$ Department of Gastroenterology, University Hospital Hairmyres, East Kilbride, UK

${ }^{4}$ The University of Edinburgh Edinburgh Medical School, Edinburgh, UK

${ }^{5}$ Gut Group, Ninewells Hospital and Medical School, Dundee, UK

${ }^{6}$ Division of Molecular and Cellular Medicine, University of Dundee School of Medicine, Dundee, UK

${ }^{7}$ Department of Gastroenterology, Glasgow Royal Infirmary, Glasgow, UK

${ }^{8}$ Department of Gastroenterology, NHSGGC, Glasgow, UK

${ }^{9}$ Dumfries and Galloway Acute Hospitals, Dumfries, UK

${ }^{10}$ Department of Gastroenterology, Dumfries and Galloway Acute Hospitals,

Dumfries, UK

${ }^{11}$ Gastroenterology, NHS Lanarkshire, Lanarkshire, Scotland

${ }^{12}$ Gastroenterology, NHS Greater Glasgow and Clyde, Glasgow, Scotland

Twitter Paul N Brennan @brennap9

Contributors TM, PB, IC, TC, TLG, MJ, MIK, VL, JS and DT were involved in basic data acquisition and analysis. TM and PB drafted the manuscript and undertook critical appraisal of document. KJS was responsible for original research design and provided input in critical manuscript writing and editing. All authors approved the final draft of manuscript. KJS accepts responsibility as guarantor of the article.

Funding The authors have not declared a specific grant for this research from any funding agency in the public, commercial or not-for-profit sectors.

Competing interests None declared.

Patient consent for publication Not applicable.

Provenance and peer review Not commissioned; externally peer reviewed.

Data availability statement All data relevant to the study are included in the article or uploaded as supplementary information.

Supplemental material This content has been supplied by the author(s). It has not been vetted by BMJ Publishing Group Limited (BMJ) and may not have been peer-reviewed. Any opinions or recommendations discussed are solely those of the author(s) and are not endorsed by BMJ. BMJ disclaims all liability and responsibility arising from any reliance placed on the content. Where the content includes any translated material, BMJ does not warrant the accuracy and reliability of the translations (including but not limited to local regulations, clinical guidelines, terminology, drug names and drug dosages), and is not responsible for any error and/or omissions arising from translation and adaptation or otherwise.

Open access This is an open access article distributed in accordance with the Creative Commons Attribution 4.0 Unported (CC BY 4.0) license, which permits others to copy, redistribute, remix, transform and build upon this work for any purpose, provided the original work is properly cited, a link to the licence is given, and indication of whether changes were made. See: https://creativecommons.org/ licenses/by/4.0/.

\section{ORCID iDs}

Paul N Brennan http://orcid.org/0000-0001-8368-1478

lain A Murray http://orcid.org/0000-0002-1795-3647

\section{REFERENCES}

1 Moon AM, Webb GJ, Aloman C, et al. High mortality rates for SARSCoV-2 infection in patients with pre-existing chronic liver disease and cirrhosis: Preliminary results from an international registry. $J$ Hepatol 2020;73:705-8.

2 Scottish government Lockdown data, 2020. Available: https://www. gov.scot/news/effective-lowdown-to-be-introduced/ [Accessed 19 Dec 2020].

3 Alcohol Change UK. Available: https://alcoholchange.org.uk/blog/ 2020/drinking-in-the-uk-during-lockdown-and-beyond [Accessed 19 Dec 2020].

4 Kim JU, Majid A, Judge R, et al. Effect of COVID-19 lockdown on alcohol consumption in patients with pre-existing alcohol use disorder. Lancet Gastroenterol Hepatol 2020;5:886-7.

5 Scottish Public Health Observatory data. Available: https://www. scotpho.org.uk/health-wellbeing-and-disease/chronic-liver-disease/ data/international-comparisons/ [Accessed 19 Dec 2020].

6 Mahmud N, Hubbard RA, Kaplan DE, et al. Declining cirrhosis hospitalizations in the wake of the COVID-19 pandemic: a national cohort study. Gastroenterology 2020;159:1134-6.

7 Gaspar R, Liberal R, Branco CC, et al. Trends in cirrhosis hospitalizations during the COVID-19 pandemic. Dig Liver Dis 2020;52:942-3.

8 Barber K, Madden S, Allen J, et al. Elective liver transplant list mortality: development of a United Kingdom end-stage liver disease score. Transplantation 2011;92:469-76.

9 Kamath PS, Wiesner $\mathrm{RH}$, Malinchoc M, et al. A model to predict survival in patients with end-stage liver disease. Hepatology 2001;33:464-70.

10 SIMD, 2020. Available: https://www.gov.scot/collections/scottishindex-of-multiple-deprivation-2020/ [Accessed 19 Dec 2020].

11 Amaddeo G, Brustia R, Allaire M, et al. Impact of COVID-19 on the management of hepatocellular carcinoma in a high-prevalence area. JHEP Rep 2021;3:100199.

12 Gandhi Met al. Impact of COVID-19 on hepatocellular carcinoma management: a multicountry and region study.

13 . Available: https://www.scotpho.org.uk/health-wellbeing-anddisease/chronic-liver-disease/data/mortality/ [Accessed 26 Feb 2021].

14 . Available: https://www.gov.scot/policies/alcohol-and-drugs/ minimum-unit-pricing/\#evaluating [Accessed 24 Mar 2021]. 
15 . Available: http://www.healthscotland.scot/media/3332/evaluatingthe-impact-of-mup-on-sales-based-alcohol-consumption-inscotland-controlled-interrupted-time-series-analyses-briefingupdated-march-2021.pdf [Accessed 24 Mar 2021].

16 Stevely Aet al. Changes in alcohol consumption in Scotland during the early stages of the COVID-19 pandemic: descriptive analysus of repeat cross-sectional survey data. Edinburgh: Public Health Scotland, 2021.

17 Li J, Liu D, Yan J, et al. Reductions in liver cirrhosis hospitalizations during the COVID-19 pandemic. Hepatol Int 2021;15:213-4.

18 Hall ME, Vaduganathan M, Khan MS, et al. Reductions in heart failure hospitalizations during the COVID-19 pandemic. J Card Fail 2020;26:462-3.

19 Bromage $\mathrm{DI}$, Cannatà A, Rind IA, et al. The impact of COVID-19 on heart failure hospitalization and management: report from a heart failure unit in London during the peak of the pandemic. Eur $J$ Heart Fail 2020;22:978-84.
20 De Filippo O, D'Ascenzo F, Angelini F, et al. Reduced rate of hospital admissions for ACS during Covid-19 outbreak in northern Italy. $N$ Engl J Med 2020;383:88-9.

21 Höner Zu Siederdissen C, Schultalbers M, Wübbolding M, et al. Impact of the COVID-19 pandemic on patients with liver cirrhosisthe experience of a tertiary center in Germany. $Z$ Gastroenterol 2021;59:954-60.

22 COVID 19 daily dashboard data. Available: https://public.tableau. com/profile/phs.covid.19\#!/vizhome/COVID-19DailyDashboard 15960160643010/Overview.-accessed [Accessed 26 Feb 2021].

23 Tapper EB, Asrani SK. The COVID-19 pandemic will have a long-lasting impact on the quality of cirrhosis care. $J$ Hepatol 2020;73:441-5.

24 Toyoda H, Huang DQ, Le MH, et al. Liver Care and Surveillance: The Global Impact of the COVID-19 Pandemic. Hepatol Commun 2020;4:1751-7.

25 Blach S, Kondili LA, Aghemo A, et al. Impact of COVID-19 on global HCV elimination efforts. J Hepatol 2021;74:31-6. 